\title{
Abnormal expression pattern of the ASPP family of proteins in human non-small cell lung cancer and regulatory functions on apoptosis through p53 by iASPP
}

\author{
SHIJUN LI ${ }^{1}$, GUANGXIA SHI ${ }^{4}$, HONG YUAN ${ }^{1}$, TAO ZHOU ${ }^{2}$, \\ QIUPING ZHANG ${ }^{3}$, HONG ZHU ${ }^{1}$ and XIAOYAN WANG ${ }^{1}$
}

Departments of ${ }^{1}$ Clinical Laboratory, ${ }^{2}$ Oncology and ${ }^{3}$ Pathology, The First Affiliated Hospital of Dalian Medical University, Dalian, Liaoning 116011; ${ }^{4}$ Department of Pathophysiology, Dalian Medical University, Dalian, Liaoning 116044, P.R. China

Received January 12, 2012; Accepted March 29, 2012

DOI: $10.3892 /$ or.2012.1778

\begin{abstract}
The p53 protein is one of the best-known tumor suppressors. Recently discovered ASPP1 and ASPP2 are specific activators and iASPP is an inhibitor of p53. In the present study, we found that of 37 NSCLC patients, p53 alterations were detected in 20 tumors (54.1\%), the mRNA expression of ASPP1 and ASPP2 was frequently dowregulated in tumor tissues, and this decreased significantly in samples expressing wild-type p53. The expression of ASPP1 and ASPP2 was downregulated and that of iASPP was upregulated in two NSCLC cell lines (the NCI-H157 cell line with altered p53 and the A549 cell line with wild-type p53). The NCI-H157 cell with higher ASPP1 and ASPP2 levels was more sensitive to cisplatin than the A549 cells with lower ASPP1 and ASPP2 levels. Downregulation of iASPP by siRNA stimulated apoptosis through p53 in two NSCLC cell lines. These findings provide new insights into the molecular mechanisms of action of the ASPP family in NSCLC and may have potent therapeutic applications.
\end{abstract}

\section{Introduction}

Lung cancer is divided into two morphological types, small cell lung cancer (SCLC) and non-small cell lung cancer (NSCLC). Lung carcinoma is currently the leading cause of cancer mortality. Therefore, many investigators are studying the molecular and biologic characteristics of lung carcinoma in an attempt to develop effective targeted therapy.

The $\mathrm{p} 53$ protein is one of the best known tumor suppressor proteins, playing a key role in apoptosis, trigger cell cycle

Correspondence to: Dr Shijun Li, Department of Clinical Laboratory, The First Affiliated Hospital of Dalian Medical University, 222 Zhongshan Road, Dalian, Liaoning 116011, P.R. China

E-mail: lishijun@dl.cn

Key words: non-small cell lung cancer, ASPP family of proteins, p53, apoptosis arrest (1), and possessing high frequency of mutations in a wide range of human cancers. In some studies, approximately $50 \%$ of NSCLC carry the $p 53$ gene alteration $(2,3)$.

The apoptosis stimulating protein of p53 (ASPP) family consists of three proteins, ASPP1, ASPP2 and iASPP. ASPP1 and ASPP2 function as tumor-suppressor genes and have been reported to interact with p53 and specifically enhance p53-induced apoptosis, but not that of the cell cycle arrest $(4,5)$. Subsequent studies further demonstrate that ASPP1 and ASPP 2 can regulate apoptosis mediated by p63 and p73 and function as common activators of p53 family members (6). The iASPP protein identified is a pro-oncogene, inhibits p53-mediated apoptosis $(7,8)$, which implies that iASPP can compete with ASPP1 and ASPP2 for binding to p53, thereby inhibiting the ability of ASPP1 and ASPP2 to stimulate the apoptosis function of $\mathrm{p} 53$.

Altered expression of the ASPP family members has been found in a variety of human cancers. The expression of ASPP1 and ASPP2 is frequently downregulated in human breast carcinomas expressing wild-type p53 (4), and in three tumor cell lines retaining wide-type p53 (9). Higher expressions of iASPP were also detected in human breast carcinomas (7). Reduced levels of ASPP2 have also been detected in colon cancer cell lines (10), and ASPP1 downregulation was observed in two mesothelioma cell lines (11). The level of ASPP1 is downregulated and that of the iASPP is upregulated in leukemia cell lines and acute leukemia tissues $(12,13)$. The expression of ASPP1 and ASPP2 was diminished in seven hepatocellular carcinoma cell lines (14).

Moreover, previous studies have shown downregulation of ASPP1 in NCI-H226 lung carcinoma cell line (11) and reduced levels of ASPP2 in lung carcinoma cell lines (10). The expression of iASPP was significantly higher in lung cancer tissues compared with the normal tissues in lung cancer (15). These results indicated that ASPP family may produce a marked effect in lung carcinoma. In this study we analyzed the correlation between the mRNA expression levels of ASPP1, ASPP2 and the status of p53 in human NSCLC samples, and the expression pattern of ASPP1, ASPP2 and iASPP in two human NSCLC cell lines (1 with wild-type p53 and 1 with altered p53). We also investigated the sensitivity of two NSCLC cell 
lines to cisplatin, as well as regulatory function on apoptosis by iASPP in NSCLC cell lines.

\section{Materials and methods}

Patient samples and immunohistochemistry. We studied a series of 37 Chinese patients with NSCLC who had undergone surgery between February and December 2007 at First Affiliated Hospital of Dalian Medical University (Dalian, Liaoning, China). There were 21 males and 16 females with a mean age of 61 years at the time of diagnosis (range, 38-75 years). Of the 37 patients, histopathologically confirmed adencarcinoma in 15 patients, 16 patients of squamous carcinoma, 2 patients with giant cell carcinoma and adencarcinoma in 4 patients for the differentiation of the squamous carcinoma. Resected tumor specimens, corresponding adjacent non-tumor and normal tissue were snap-frozen and stored at $-80^{\circ} \mathrm{C}$.

The expression of mutant p53 protein in the samples was detected by immunohistochemistry. The cancer, paracarcinomatous and normal tissue samples were fixed with formalin for $9 \mathrm{~h}$, paraffin-embedded, and blocks were cut at $4 \mu \mathrm{m}$ thick sections. The sections were treated with $3 \% \mathrm{H}_{2} \mathrm{O}_{2}$ for $10 \mathrm{~min}$ at room temperature to inactivate endogenous peroxidase activity. Subsequently sections were incubated with p53 monoclonal antibody for $60 \mathrm{~min}$ and with a peroxidase goat anti-mouse IgG complex (Maxim, Fuzhou, China) for $15 \mathrm{~min}$ at room temperature. Each section was added freshly prepared DAB reagent and observed under the microscope. The sections were then counterstained with hematoxylin, dehydrated, transparent and blocked with neutral gum.

Computational analysis and software. Genomic DNA, cDNA and protein sequences of ASPP family were obtained from the NCBI GenBank (http://www.ncbi.nlm.nih.gov) and UCSC Genome Browser home page (http://www.genome.ucsc.edu), and the analysis of homologous identity was performed using Jellyfish3.3 (http://www.jellyfishsoftware.com) and BLAST (http://blast.ncbi.nlm.nih.gov).

Cell lines and cell culture. The human non-small cell lung squamous carcinoma A549 cells and human non-small cell lung adencarcinoma NCI-H157 cells were obtained from the Cell Centre of School of Basic Medicine Peking Union Medical College (Beijing, China) and maintained in RPMI1640 medium supplemented with $10 \%(\mathrm{v} / \mathrm{v})$ fetal bovine serum (Hyclone). Two cell lines were incubated at $37^{\circ} \mathrm{C}$ in a humidified atmosphere with $5 \% \mathrm{CO}_{2}$.

$R N A$ preparation, reverse transcription and quantitative realtime qPCR. Total RNA was extracted from A549, NCI-H157 cell lines and various tissue specimens using RNAiso reagent (Takara). The RNA was reverse-transcribed into cDNA with a PrimeScript II First Strand cDNA Synthesis kit (Takara). Quantitative real-time PCR was carried out in the LightCycle real-time PCR (Roche) using SYBR Premix Ex Taq ${ }^{\mathrm{TM}}$ kit for Perfect Real-Time (Takara). All the real-time PCR primers used are listed in Table I.

MTT assay and cell growth determination. Cell growth was measured by 3-(4,5)-dimethylthiazol(-2-y1)-3,5-di-
Table I. Primers used in qRT-PCR analysis.

\begin{tabular}{ll}
\hline Primer & \multicolumn{1}{c}{ Sequence $\left(5^{\prime} \rightarrow 3^{\prime}\right)$} \\
\hline ASPP1 & F: CAGCAG CA GATGTTGGT TGC \\
& R: TCCTGGGCTTCAACTCGTTC \\
ASPP2 & F: CAGTTGGCCCTGATTGGAG \\
& R: ACGGACGCACTTTCTTCTCTTT \\
iASPP & F: CAGCCACAATCACAACCACAGC \\
& R: TTCATCCACATCGCCAGCCTC \\
$\beta$-actin & F: CCACGAAACTACCTTCAACTCC \\
& R: GTGATCTCCTTCTGCATCCTGT
\end{tabular}

phenyltetrazolium bromide (MTT) assay (Sigma) following the instructions of the manufacturer. Cells were plated into 96-well plates and treated with 0.75, 1.5, 3.0,6.0, $12.0 \mu \mathrm{g} / \mathrm{ml}$ of cisplatin. After $24 \mathrm{~h}$, MTT reagent $(5 \mu \mathrm{g} / \mu \mathrm{l})$ was added (15 $\mu \mathrm{l} /$ well) and incubated at $37^{\circ} \mathrm{C}$ for $4 \mathrm{~h}$. The reaction was stopped with addition of $150 \mu \mathrm{l}$ DMSO and the optical density was determined at OD $570 \mathrm{~nm}$ on a multi-well plate reader. Each sample was done in triplicate and repeated three times.

Protein preparation and western blotting. Total protein of A549 and NCI-H157 cells was extracted with RIPA lysis buffer $(150 \mathrm{mM} \mathrm{NaCl}, 25 \mathrm{mM}$ Tris- $\mathrm{HCl} \mathrm{pH} 7.6,1 \% \mathrm{NP}-40$, $1 \%$ sodium deoxycholate, $1 \%$ SDS and $1 \%$ protease inhibitor cocktail). The cells were harvested, washed and lysed in cold RIPA buffer.

Total proteins were resolved using 8\% SDS-PAGE and transferred to an NC-membrane (Millipore, Darmstadt, Germany). Western blotting was performed according to standard protocols. To analyze ASPP family protein levels, we generated three monoclonal antibodies against the $120-\mathrm{kDa}$ ASPP1 protein (1,090 amino acids), 126-kDa ASPP2 protein (1,128 amino acids) and 89-kDa iASPP protein (828 amino acids). The secondary antibody was goat anti-mouse IgG conjugated to horseradish peroxidase (1:3000, Pierce). Immunoblots were visualized on photographic film using the ECL kit (Pierce), following the method provided by the supplier. For the loading control, anti- $\beta$-actin monoclonal antibody (1:5000, Sigma) was used.

Detection of apoptosis. Cell morphology and apoptosis were analyzed by a transmission electron microscope. Trypsin digestion of cells from a single cell suspension, at low-speed centrifugation, and placement in ice-cold $15 \%$ glutaraldehyde and $1 \%$ osmium acid, then double-fix-embedded, cut to $70-\mathrm{nm}$ thick sections. Toluidine staining was applied, light microscopy positioning, and transmission electron microscopy observation of cell ultrastructure followed.

RNA interference. RNA interference was performed by using the synthetic siRNA duplexes (Takara). Two synthetic siRNA duplexes (si1-iASPP and si2-iASPP) targeting iASPP cDNA sequences 5'-ACAGTACCAGCAGATCATC-3' and 5'-TTAC AAGGTCTCTCCCTCTG-3' were used to inhibit iASPP 
A

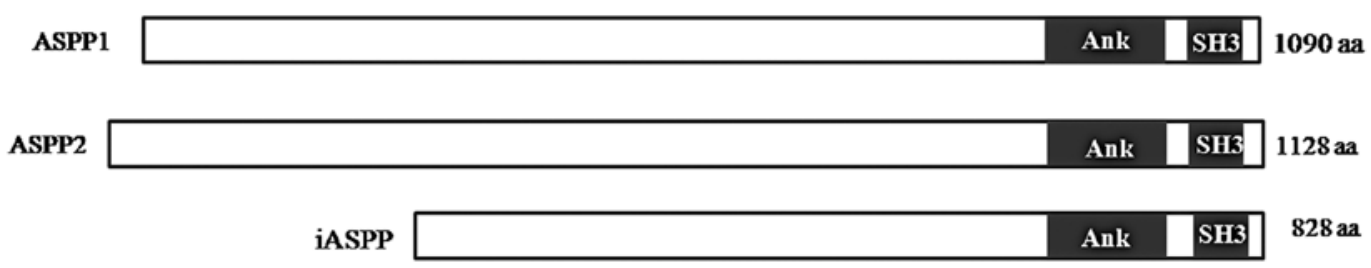

B

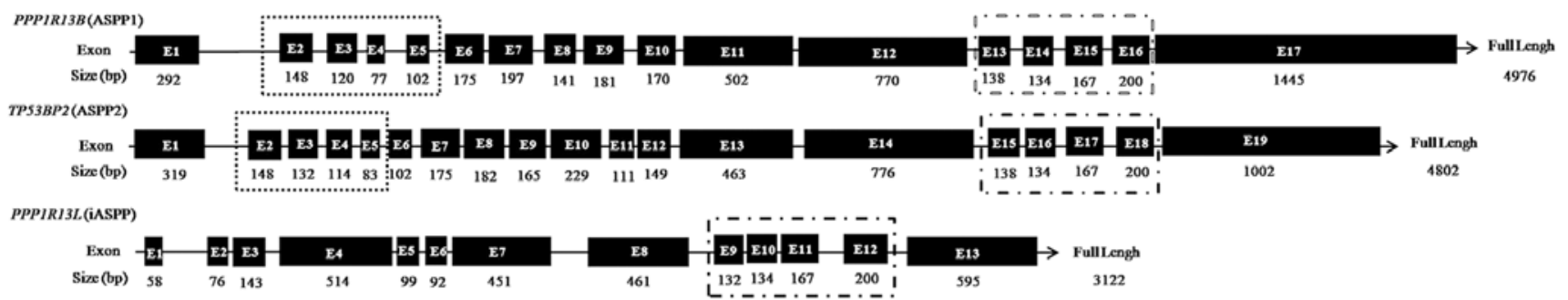

Figure 1. Structure analysis of human ASPP family. (A) Schematic representation of the ASPP family of proteins, the dark boxes indicate protein structure domain (Ank, ankyin repeats; SH3, Src homology 3 domain). (B) Exon composition and similar relationship among the three genes of the ASPP family, dotted line boxes indicate highly similar exons.

Table II Similarity comparison of the human ASPP family of proteins ASPP1, ASPP2, and iASPP.

\begin{tabular}{lcc}
\hline & Protein $(\%)$ & mRNA $(\%)$ \\
\hline ASPP1 vs. ASPP2 & 47.5 & 53.6 \\
ASPP1 vs. iASPP & 22.5 & 41.9 \\
ASPP2 vs. iASPP & 21.5 & 41.7 \\
\hline
\end{tabular}

mRNA expression. The siControl (5'-AGCGTACCCTACAGA TCAA-3') was a scrambled sequence with no homology to any known gene and used as a negative control. Cells were transfected with $50 \mathrm{nM}$ of siRNA using Lipofectamine 2000 (Invitrogen) according to the manufacturer's instruction.

Statistical analysis. The analyses were carried out using SPSS 13.0 for Windows software. Data are presented as mean \pm SD, and comparison between the two groups used single factor analysis of variance. A $\mathrm{P}<0.05$ was considered statistically significant.

\section{Results}

Comparative analysis of the ASPP family. The human ASPP family is composed of three proteins, ASPP1, ASPP2 and iASPP. iASPP protein identified is by far the most evolutionary conserved member of ASPP family, and the only member identified in Caenorhabditis elegans (7). Mammals, including mice and humans, have evolved two additional members, ASPP1 and ASPP2 (4). Three proteins share structure similarity in C-termini which contains their signature sequence of Ankyin repeats and SH3 domain (Fig. 1A). Study confirmed that the ASPP family members bind the core domain of $\mathrm{p} 53$ through Ankyin repeats and SH3 domain. ASPPl is a 1,090- amino acid protein, encoded by gene PPPIRI3B, and ASPP2 consists of 1,128 amino acids, encoded by $p 53 B P 2$ gene. In human, PPPIR13L gene encodes the iASPP, complete iASPP composed of 828 amino acids. Protein sequence analysis showed $47.5 \%$ similarity between ASPP1 and ASPP2 protein (Table II).

Gene PPPIRI3B, p53BP2 and PPPIR13L is located in 14q32.33, 1q41, 19q13.32 chromatin, respectively. The cDNAs of PPPIRl $3 B$ and $p 53 B P 2$ are 4,976 bp and 4,802 bp long, respectively, with each consisting of 17 exons and the other consisting of 19 exons. PPPI R13L cDNA has a full length of $3,122 \mathrm{bp}$ and consists of 13 exons (Fig. 1B). Sequence analysis revealed that the ASPP1 and ASPP2 mRNA sequences share similarity with iASPP (>40\%), ASPP1 and ASPP2 with higher similarity ( $>50 \%$ ) (Table II), ASPP1 is the homology analogues of ASPP2.

Expression of mut-p53 protein and ASPP1, ASPP 2 mRNA in NSCLC tissues and correlation of the expression of ASPPI, ASPP 2 with the development of NSCLC. To investigate the possible connection between ASPP1, ASPP2 and p53 in human NSCLC, we first analyzed the expression of the mut-p53 protein in cancer tissues and normal lung tissues by carrying out immunohistochemical staining. Mut-p53 protein located in the nucleus, the nuclei appeared brown for the positive expression of mut-p53 (Fig. 2A). We found that in 20 out of 37 patient cancer tissues (54.1\%) showed mut-p53 protein positivity, of which adenocarcinoma in 9 patients, squamous cell carcinoma in 10 patients and giant cell carcinoma 1 patient, the mut-p53 expression between adenocarcinoma and squamous cell carcinoma was not significantly different $(\mathrm{P}>0.05)$. Normal lung tissues in 2 patients were positive for mut-p53 protein, the positive rate was $5.4 \%$ (Fig. 2B).

To confirm the expression of ASPP1 and ASPP2 mRNAs, we carried out real-time qPCR. In cancer, paracarcinomatous and normal lung tissues, the expression of ASPP1 and ASPP2 
A
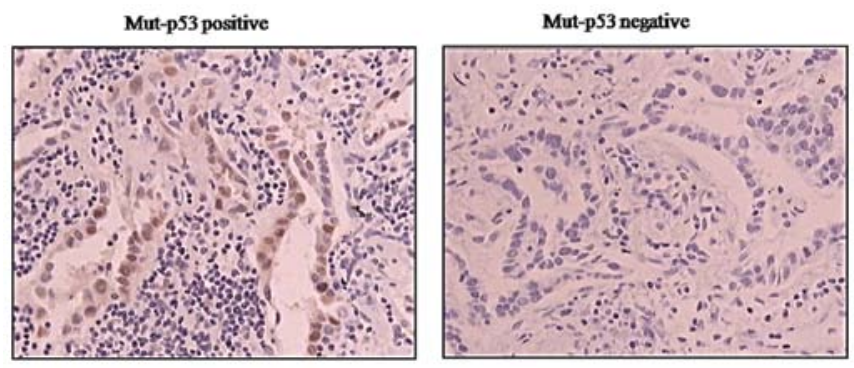

C

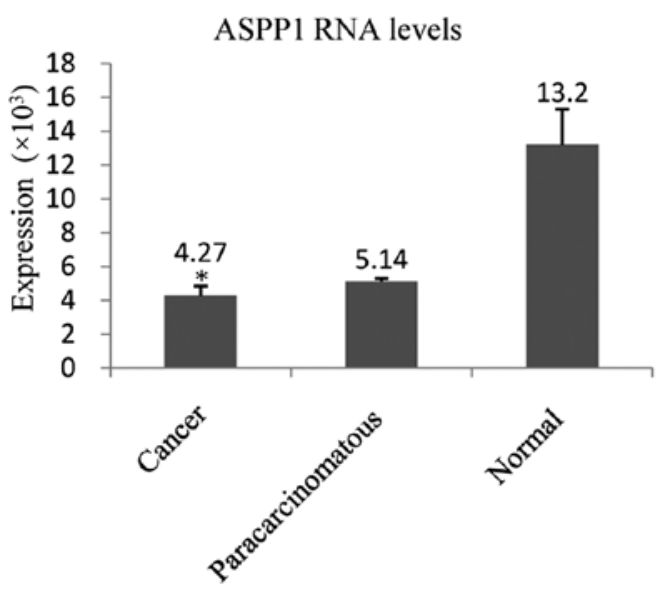

D

ASPP1 RNA levels

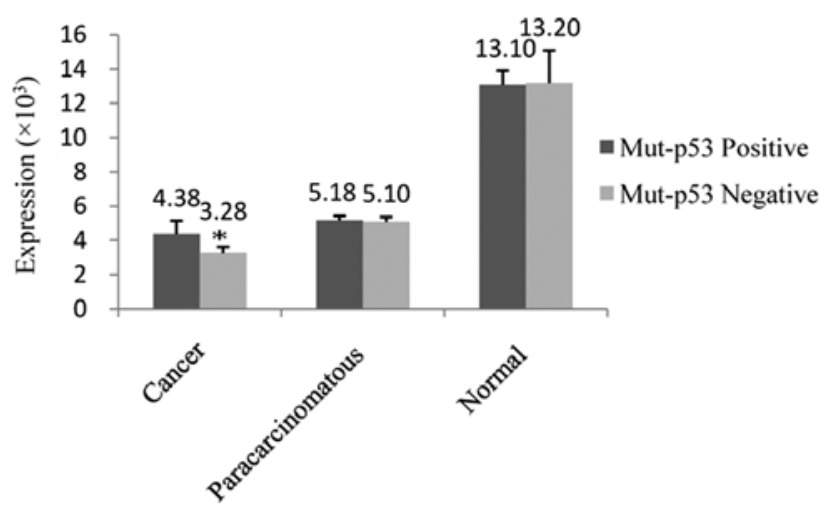

$$
\begin{aligned}
& \text { Mut-p53 protein } \\
& \text { expression }
\end{aligned}
$$
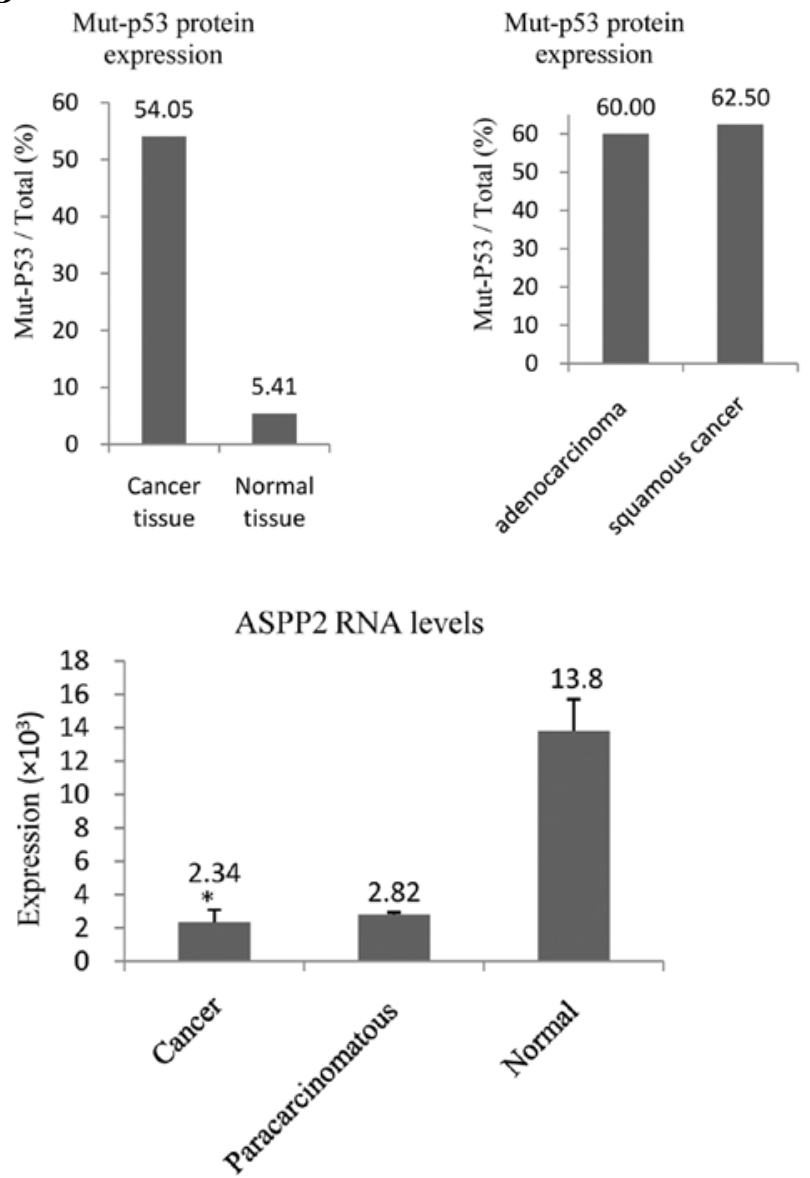

ASPP2 RNA levels

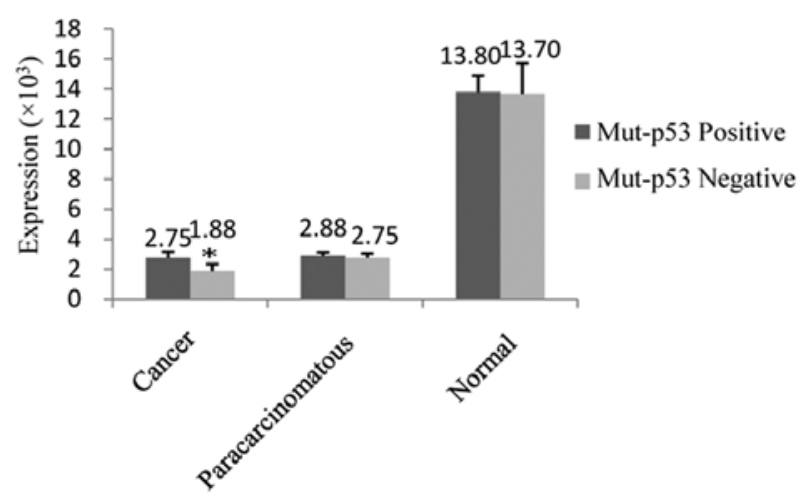

Figure 2. Expression of mut-p53 protein and ASPP1, ASPP2 mRNA in NSCLC tissues. (A) Mut-P53-positive protein was determined by immunohistochemistry. (B) The ratio of the expressed mut-p53 protein cases to the total cases was detected to analyze the p53 protein status in NSCLC patients. (C) The RNA expression of ASPP1 and ASPP2 was analyzed using quantitative real-time RT-PCR in cancer tissues, paracarcinomatous tissues and normal tissues. Statistical analysis showed lower RNA level of ASPP1 and ASPP2 in cancer tissues than normal tissues ("P<0.05). Error bars represent the standard errors of three independent experiments. (D) Analysis of the RNA level of ASPP1 and ASPP2 between mut-P53-positive group and mut-P53-negative group. Mut-P53 negative samples showed lower expression of ASPP1 and ASPP2 than mut-P53-positive samples in cancer tissues ("P $<0.05)$. Error bars represent the standard errors of three independent experiments.

mRNA showed a gradual increasing trend. The mRNA levels of ASPP1 and ASPP2 in cancer tissues was lower than the paracarcinomatous and normal lung tissues $(\mathrm{P}<0.05)$ (Fig. 2C).

We also analyzed the mRNA expression of ASPP1 and ASPP2 between mut-p53 positive group and mut-p53-negative group. We found that the ASPP1 and ASPP2 mRNA levels were lower in mut-p53-negative cancer tissues than in mut-p53positive cancer tissues $(\mathrm{P}<0.05)$ (Fig. 2D), demonstrating that human NSLC in expression of wild-type p53 protein may be associated with the decreased mRNA levels of ASPP1 and ASPP2. 
A
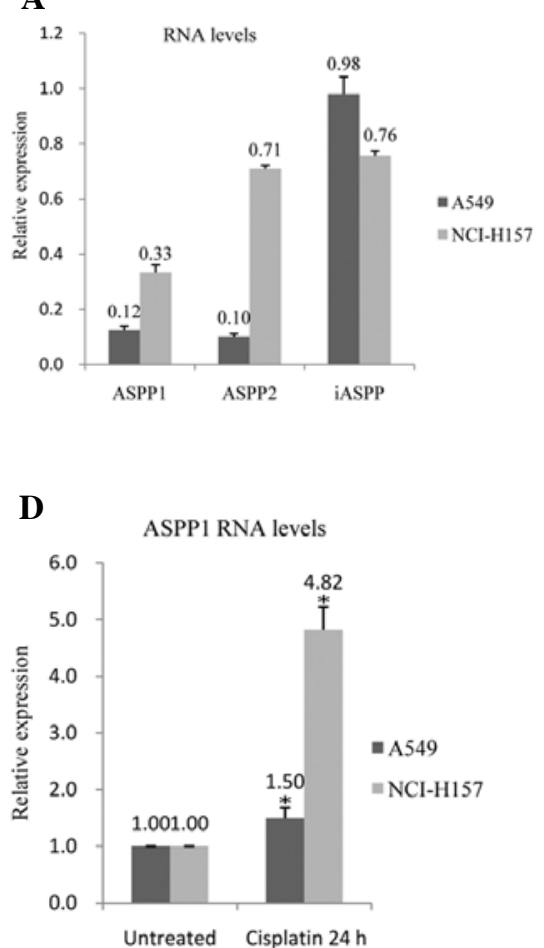

B

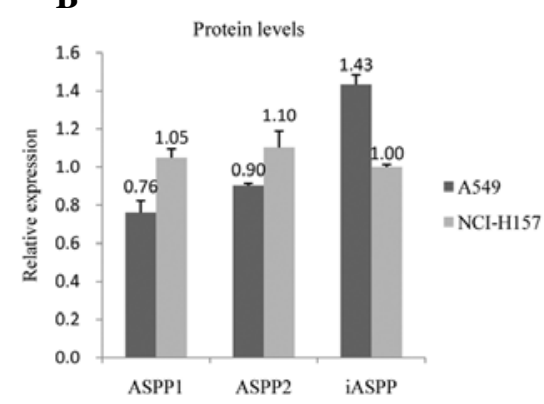

ASPP2 RNA levels

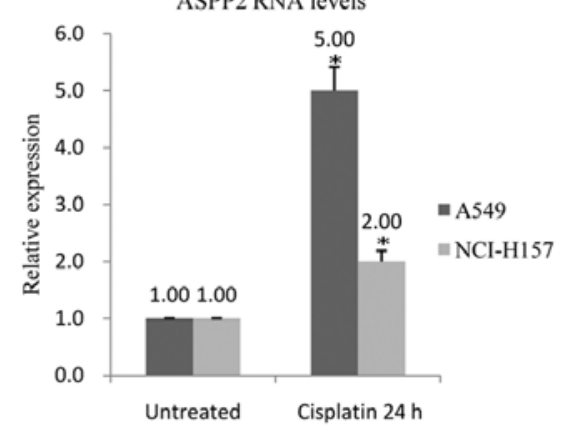

C

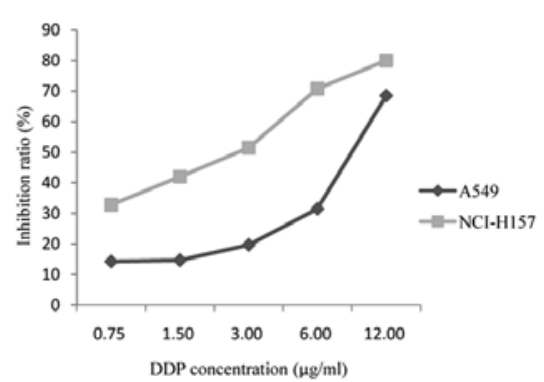

iASPP RNA levels

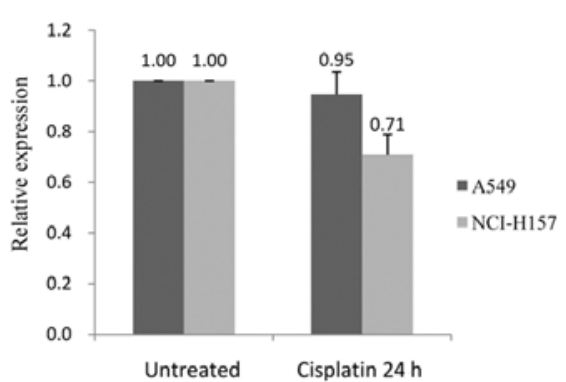

$\mathbf{E}$
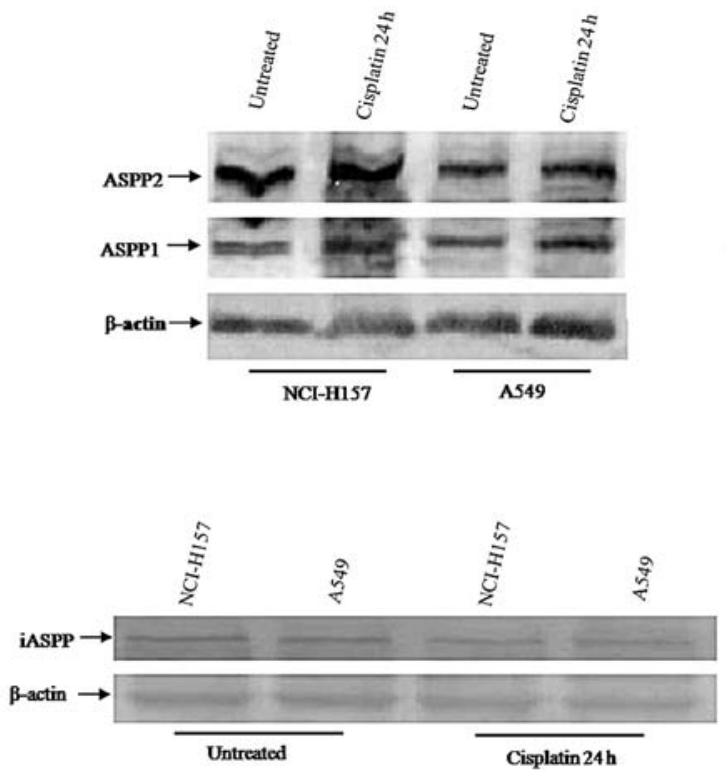

F
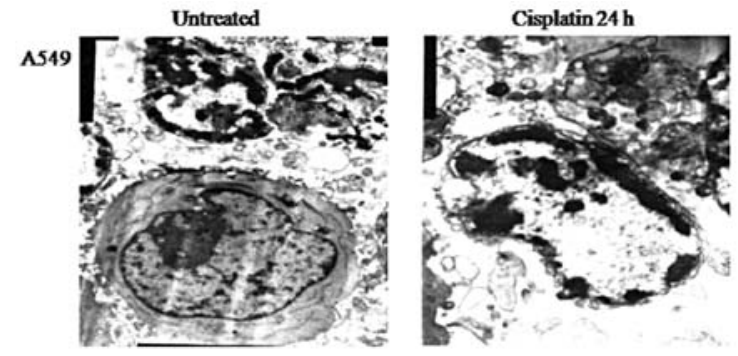

NCI-H157
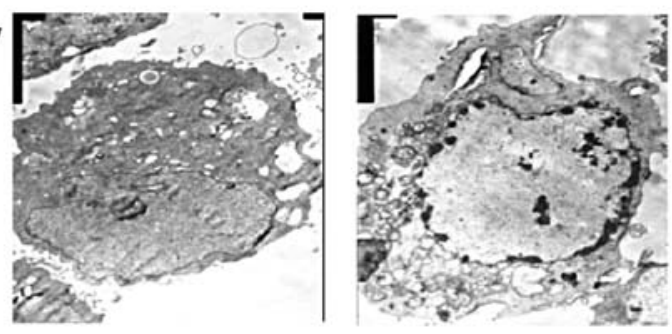

Figure 3. Expression of ASPP family mRNA and protein in NSCLC cell lines, sensitivity to cisplatin of NSCLC cells, and changes in ASPP1, ASPP2 and iASPP mRNA and protein levels after cisplatin treatment. (A) The RNA expression of ASPP family was analyzed using quantitative real-time RT-PCR in A549 and NCI-H157 cell lines, with the $\beta$-actin gene as an internal control. Error bars represent the standard errors of three independent experiments. (B) Western blot analysis of ASPP family protein expression in A549 and NCI-H157 cell lines, with the $\beta$-actin protein as an internal control. (C) Cells were treated with cisplatin $(0.75,1.5,3.0,6.0,12.0 \mu \mathrm{g} / \mathrm{ml})$ and cell viability was determined by MTT assay; the IC50 value of A549 cell was $10.5 \mu \mathrm{g} / \mathrm{ml}$ and the IC50 value of NCI-H157 cells was $3.7 \mu \mathrm{g} / \mathrm{ml}$. (D) Quantitative real-time PCR analysis of the RNA levels of ASPP1, ASPP2, and iASPP after cisplatin treatment for $24 \mathrm{~h}$ in A549 and NCI-H157 cells, with the $\beta$-actin gene as an internal control. Error bars represent the standard errors of three independent experiments. (E) Western blot analysis of total cell lysates from cisplatin treatment in A549 and NCI-H157 cells for $24 \mathrm{~h}$ using anti-ASPP1 antibodies, anti-ASPP2 antibodies and antiiASPP antibodies with the $\beta$-actin protein as a loading control. (F) Cell morphology and apoptosis were analyed by transmission electron microscopy before and after cisplatin treatment.

Analysis of ASPP family mRNA and protein level in NSCLC cell lines. We analyzed RNA and protein expressions of ASPP1, ASPP2 and iASPP with quantitative real-time PCR and western blot analysis in two NSCLC cell lines (A549 cell line with wild-type p53 and NCI-H157 cell line with altered p53). Analysis showed that the mRNA level of ASPP1 and ASPP2 was downregulated and that of the iASPP was upregulated in two cell lines, and specifically showed remarkably low expression of ASPP1, and ASPP2, but relatively high expression of iASPP in A549 cell (Fig. 3A). The protein expression 
A

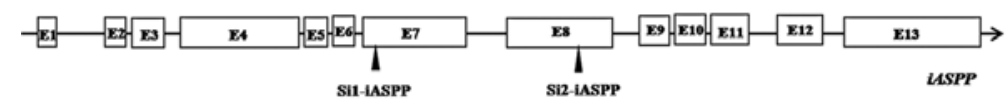

C

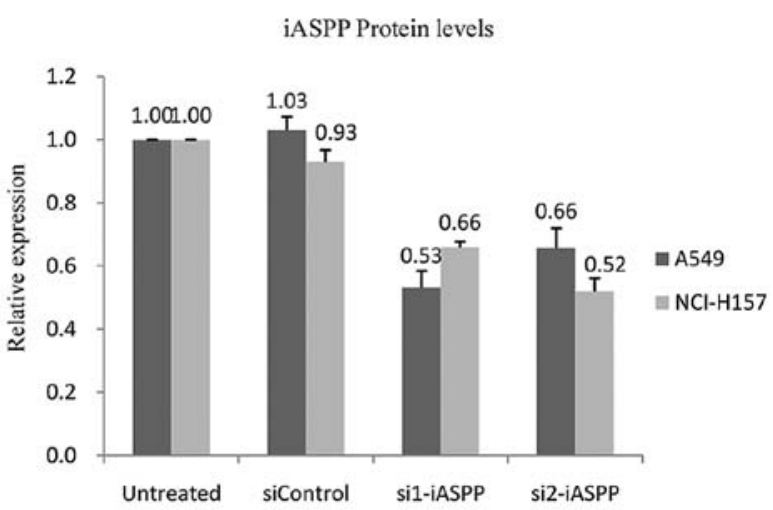

B

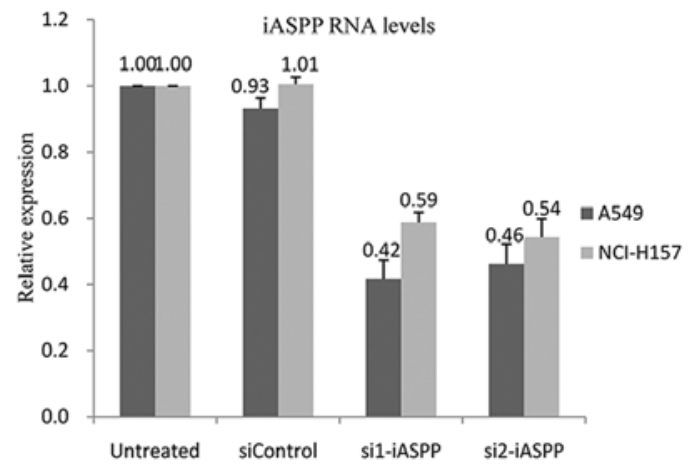

D
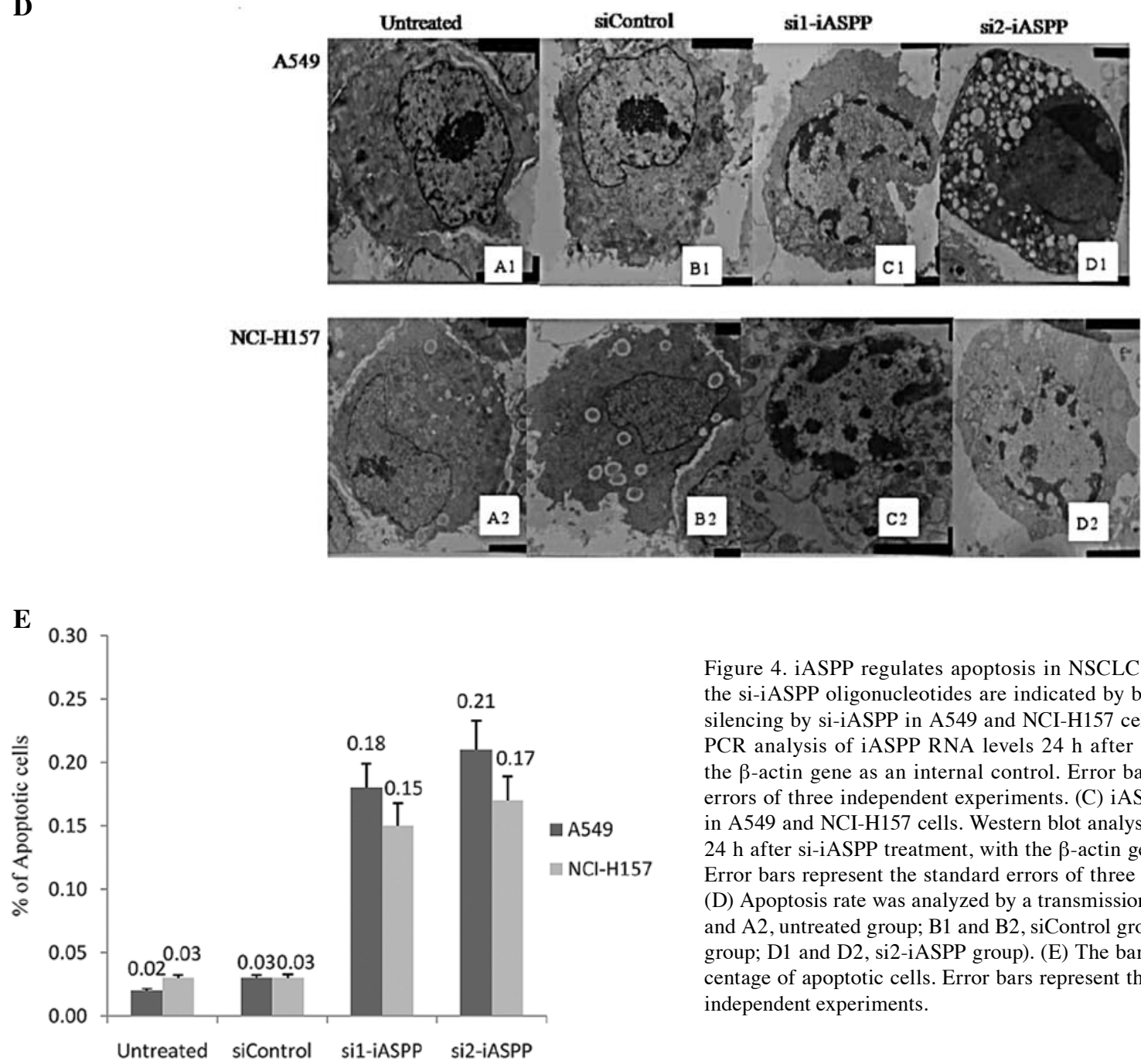

Figure 4. iASPP regulates apoptosis in NSCLC cell. (A) The position of the si-iASPP oligonucleotides are indicated by black triangles. (B) iASPP silencing by si-iASPP in A549 and NCI-H157 cells. Quantitative real-time PCR analysis of iASPP RNA levels $24 \mathrm{~h}$ after si-iASPP treatment, with the $\beta$-actin gene as an internal control. Error bars represent the standard errors of three independent experiments. (C) iASPP silencing by si-iASPP in A549 and NCI-H157 cells. Western blot analysis of $i A S P P$ protein levels $24 \mathrm{~h}$ after si-iASPP treatment, with the $\beta$-actin gene as an internal control. Error bars represent the standard errors of three independent experiments. (D) Apoptosis rate was analyzed by a transmission electron microscope (A1 and A2, untreated group; $\mathrm{B} 1$ and $\mathrm{B} 2$, siControl group; $\mathrm{C} 1$ and $\mathrm{C} 2$, si1-iASPP group; D1 and D2, si2-iASPP group). (E) The bar graphs represent the percentage of apoptotic cells. Error bars represent the standard errors of three independent experiments.

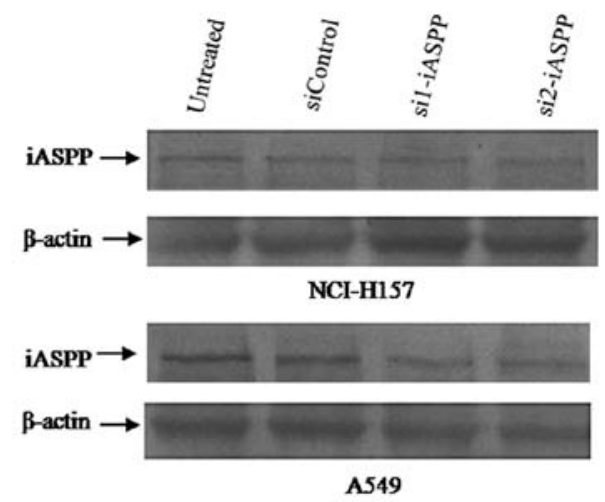


of ASPP1 and ASPP2 was fewer than iASPP in A549 cell, coinciding with their mRNA expressions. In NCI-H157 cell, there was no significant difference in protein expression among these three proteins (Fig. 3B).

To explore the regulation action of ASPP family in NSCLC cells, we treated cells with cisplatin and evaluated the cell viability, the changes in ASPP1, ASPP2 and iASPP mRNA and protein levels, and apoptosis.

We used the MTT assay to evaluate the growth inhibitory effect of cisplatin on A549 and NCI-H157 cell lines and calculated the IC50 values. Cisplatin inhibited cell growth in a concentration-dependent manner in two cell lines. The IC50 value for the A549 cells was $10.5 \mu \mathrm{g} / \mathrm{ml}$ and for NCI-H157 cell was $3.7 \mu \mathrm{g} / \mathrm{ml}$ (Fig. 3C). As demonstrated in Fig. 3C, the NCI-H157 cell line expressing high levels of ASPP1 and ASPP2 showed higher sensitivity to cisplatin.

The expression of ASPP1, and ASPP2 was significantly increased at the RNA and protein level after cisplatin treatment, whereas the expression of iASPP was only slightly decreased in A549 and NCI-H157 cell (Fig. 3D and E).

To confirm that cisplatin caused apoptosis, we observed cell morphology by a transmission electron microscope before and after cisplatin treatment. After cisplatin treatment organelle swelling was seen, as well as membrane retraction, irregular shrinkage, apoptotic cell appearance, and chromatin gathering under the nuclear membrane or crescent-shaped distribution (Fig. 3F).

Regulatory function on apoptosis by iASPP in NSCLC cell lines. To investigate the effects of iASPP on apoptosis, two transcript-specific small interfering RNAs (siRNAs) specifically targeting exon 7 of iASPP (si1-iASPP) and exon 8 of iASPP (si2-iASPP) against iASPP were designed and transfected into NSCLC cells (Fig. 4A). The mRNA and protein levels of iASPP were initially characterized after knockdown by si1-iASPP and si2-iASPP in A549 and NCI-H157 cell lines. The results indicated that the si1-iASPP and si2-iASPP both caused visible reductions in iASPP mRNA and protein levels (Fig. 4B and C). The si1-iASPP and si2-iASPP oligonucleotides were designed to target the sequences which do not overlap with ASPP1, and ASPP2, to avoid cross silencing.

We examined the cell morphology and cell count using transmission electron microscopy before and after the si1-iASPP and si2-iASPP treatment. The ratio of apoptotic to total cells was measured to analyze the apoptosis level. After the knockdown of iASPP, apoptotic cells were seen, the apoptosis rates were remarkably increased (Fig. 4D) in the two cell lines, suggesting that high expression of iASPP may inhibit apoptosis in NSLC cell lines.

\section{Discussion}

Our study in 37 NSCLC samples (20 of these samples express mutant p53 while the other 17 express wild-type p53) showed that the mRNA expression of ASPP1 and ASPP2 in lung tumor tissues was lower than in normal lung tissues. Thus, it was indicated that the downregulation of ASPP might be a characteristic of NSCLC tumors. Moreover, we also found the expression levels of ASPP1, and ASPP2 in tumor tissues with wild-type p53 was lower than that with mutant p53, suggested that the decreased expression of ASPP1 and ASPP2 caused the wild-type p53 to fail its role as a tumor suppressor in NSCLC.

We found that the level of ASPP1 and ASPP2 was downregulated and that of the iASPP was upregulated in two NSCLC cell lines with different genetic background, A549 cell line expressed wild-type p53 and NCI-H157 cell line expressed mutant $\mathrm{p} 53$.

Our results suggested ASPP family expression level was not associated with the p53 status in the two NSCLC cell lines, abnormal ASPP family expression could be an important step in the formation of human lung neoplasms and might be a useful molecular marker for the diagnosis of NSCLC.

The present study is the first report of downregulation of ASPP1 and ASPP2 in tumor tissues and a cell line and upregulation of iASPP in a tumor cell line with mutant p53.

We thus examined the sensitivity of these two cell lines to the DNA-damaging agent cisplatin and found that two cell lines were sensitive to cisplatin. The NCI-H157 cell line with high expression level of ASPP1, ASPP2 showed higher sensitivity for cisplatin. Upon treatment with cisplatin, the expression of ASPP1, and ASPP2 at mRNA and protein levels were significantly increased, whereas the expression of iASPP was only slightly decreased in the two cell lines. These observations suggested a possibility that expression of ASPP1 and ASPP2 might be correlated with the sensitivity to the DNA damaging agent. In A549 cell line with wild-type p53, upregulation of ASPP1 and ASPP2 expression by cisplatin should enhance the apoptotic function of p53 and profit tumor therapy. However, in NCI-H157 cell lines with mutant p53, the functions of upregulated ASPP1 and ASPP2 expression are unknown and need further study.

In the present study, we also demonstrated that iASPP is overexpressed in two human NSCLC cell lines, and siRNA mediated downregulation of iASPP results in stimulation of apoptotic function of p53. The function on apoptosis by iASPP was more significant in the A549 cell line, suggesting iASPP plays important roles in NSCLC and maybe a target for lung cancer therapy associated with the p53 pathway.

\section{Acknowledgements}

This study was supported by the Natural Science Foundation of Liaoning Province (grant no. 20092167).

\section{References}

1. Rowan S, Ludwig RL, Haupt Y, Bates S, Lu X, Oren M and Vousden KH: Specific loss of apoptotic but not cell-cycle arrest function in a human tumor derived p53 mutant. EMBO 15: 827-838, 1996.

2. Tammemagi MC, McLaughlin JR and Bull SB: Meta-analyses of p53 tumor suppressor gene alterations and clinicopathological features in resected lung cancers. Cancer Epidemiol Biomarkers Prev 8: 625-634, 1999.

3. Mitsudomi T, Hamajima N, Ogawa $M$ and Takahashi T: Prognostic significance of p53 alterations in patients with non-small cell lung cancer: a meta-analysis. Clin Cancer Res 6: 4055-4063, 2000.

4. Samuels-Lev Y, O'Connor DJ, Bergamaschi D, Trigiante G, Hsieh JK, Zhong S, Campargue I, Naumovski L, Crook T and $\mathrm{Lu}$ X: ASPP proteins specifically stimulate the apoptotic function of p53. Mol Cell 8: 781-794, 2001. 
5. Vives V, Su J, Zhong S, Ratnayaka I, Slee E, Goldin R and Lu X: ASPP2 is a haploinsufficient tumor suppressor that cooperates with p53 to suppress tumor growth. Genes Dev 20: 1262-1267, 2006.

6. Bergamaschi D, Samuels Y, Jin B, Duraisingham S, Crook T and Lu X: ASPP1 and ASPP2: common activators of p53 family members. Mol Cell Biol 24: 1341-1350, 2004.

7. Bergamaschi D, Samuels Y, O'Neil NJ, Trigiante G, Crook T, Hsieh JK, O'Connor DJ, Zhong S, Campargue I, Tomlinson ML, Kuwabara PE and Lu X: iASPP oncoprotein is a key inhibitor of p53 conserved from worm to human. Nat Genet 33: 162-167, 2003.

8. Lettre G, Kritikou EA, Jaeggi M, Calixto A, Fraser AG, Kamath RS, Ahringer J and Hengartner MO: Genome-wide RNAi identifies p53-dependent and -independent regulators of germ cell apoptosis in C. elegans. Cell Death Differ 11: 1198-1203, 2004.

9. Liu ZJ, Lu X, Zhang Y, Zhong S, Gu SZ, Zhang XB, Yang X and Xin HM: Downregulated mRNA expression of ASPP and the hypermethylation of the 5'-untranslated region in cancer cell lines retaining wild-type p53. FEBS Lett 579: 1587-1590, 2005.

10. Mori T, Okamoto H, Takahashi N, Ueda R and Okamoto T: Aberrant overexpression of 53BP 2 mRNA in lung cancer cell lines. FEBS Lett 465: 124-128, 2000.
11. Mori S, Ito G, Usami N, Yoshioka H, Ueda Y, Kodama Y, Takahashi M, Fong KM, Shimokata K and Sekido Y: p53 apoptotic pathway molecules are frequently and simultaneously altered in non-small cell lung carcinoma. Cancer 100: 1673-1682, 2004.

12. Liu ZJ, Zhang Y, Zhang XB and Yang X: Abnormal mRNA expression of ASPP members in leukemia cell lines. Leukemia 18: 880, 2004.

13. Zhang X, Wang M, Zhou C, Chen S and Wang J: The expression of iASPP in acute leukemias. Leuk Res 29: 179-183, 2005.

14. Zhao J, Wu GB, Bu FF, Lu B, Liang A, Cao L, Tong X, $\mathrm{Lu} \mathrm{X}, \mathrm{Wu} \mathrm{MC}$ and Guo YJ: Epigenetic silence of ankyrin-repeat-containing, SH3-domain-containing, and proline-rich-region-containing protein 1 (ASPP1) and ASPP2 genes promotes tumor growth in hepatitis B virus-positive hepatocellular carcinoma. Hepatology 51: 142-153, 2010.

15. Chen JF, Xie F, Zhang LJ and Jiang WG: iASPP is over-expressed in human non-small cell lung cancer and regulates the proliferation of lung cancer cells through a p53 associated pathway. BMC Cancer 10: 694, 2010 